Cinémas

Revue d'études cinématographiques

Journal of Film Studies

\title{
Musicalité ou oralité? (Réflexions d'un cinéaste qui voulait " faire comme un musicien »)
}

\section{Pierre Hébert}

Volume 3, numéro 1, automne 1992

Cinéma et Musicalité

URI : https://id.erudit.org/iderudit/1001179ar

DOI : https://doi.org/10.7202/1001179ar

Aller au sommaire du numéro

Éditeur(s)

Cinémas

ISSN

1181-6945 (imprimé)

1705-6500 (numérique)

Découvrir la revue

Citer cet article

Hébert, P. (1992). Musicalité ou oralité? (Réflexions d'un cinéaste qui voulait "faire comme un musicien »). Cinémas, 3(1), 43-63.

https://doi.org/10.7202/1001179ar

\section{Résumé de l'article}

Les correspondances entre la musique et les images abstraites du cinéma d'animation ont été historiquement marquées par la tendance à créer des musiques visuelles. En réfléchissant sur cette histoire, en partant aussi de ses expériences personnelles, le cinéaste Pierre Hébert montre ici que l’image animée peut avoir un autre rapport avec le donné musical que celui de ces musiques visuelles. 


\title{
Musicalité ou oralité? (Réflexions d'un cinéaste qui voulait «faire comme un musicien»)
}

\section{Pierre Hébert}

\begin{abstract}
RÉSUMÉ
Les correspondances entre la musique et les images abstraites du cinéma d'animation ont été historiquement marquées par la tendance à créer des musiques visuelles. En réfléchissant sur cette histoire, en partant aussi de ses expériences personnelles, le cinéaste Pierre Hébert montre ici que l'image animée peut avoir un autre rapport avec le donné musical que celui de ces musiques visuelles.
\end{abstract}

\section{ABSTRACT}

Correspondences between the music and the abstract images of animation cinema have historically been marked by the tendancy to create visual music. Using his own personal experience as a starting point for historical reflection, the filmmaker Pierre Hébert shows that there are other ways to link the animated image to the music than that of visual music.

Musicalité du cinéma : cette expression, sous des dehors un peu poétiques qui font rêver, pointe du doigt vers une zone singulièrement problématique du cinéma, celle de sa spécificité, vers un trouble dans sa définition même. En effet, si on veut prendre cette expression au sérieux, la musicalité du cinéma doit être fondée sur l'hypothèse que les principes musicaux d'organisation auraient une certaine forme de pertinence dans le 
cinéma. Étant donné la cohabitation, dans le cinéma, d'éléments visuels et sonores (dont la musique), la question est plus complexe qu'elle ne paraît à première vue. Elle pose le problème d'un rapport d'extériorité entre la musique et le cinéma pris globalement en tant qu'arts autonomes, alors que la musique fait elle-même partie des éléments constituants du cinéma dans le cadre d'un rapport interne image/son. Les deux niveaux de relation ne peuvent être dissociés.

Bien qu'il s'avère impossible d'éviter la dimension théorique de cette question, le point de vue adopté ici est celui d'un cinéaste qui, au cours des années, a été et est encore sollicité par une vision musicale du cinéma et qui, selon les moments, a apporté des réponses diverses, voire contradictoires, à cette sollicitation. Vision qui, de plus, se situe dans une tradition cinématographique bien précise et assez marginale, dont l'examen ne peut prétendre épuiser le sujet. Cela s'est passé en deux temps : d'abord, de 1964 à 1971 j'ai réalisé une série de films abstraits à forte orientation musicale, période de jeunesse que j'avais un peu oubliée et sur laquelle le présent texte m'a forcé à revenir; puis, à partir de 1983, je me suis engagé dans un travail intensif avec des musiciens, qui a largement débordé le cadre strict de la production de films pour trouver des expressions scéniques parfois inusitées. Travail sans réserve avec les musiciens mais non sans ambivalence en ce qui concerne le rapport avec la musique. Le présent texte est l'expression de cette ambivalence.

Après quelques rappels historiques d'ordre général, je reviendrai plus en détail sur ces deux phases de ma pratique personnelle, que je ne prétendrai pas exposer pour elles-mêmes à titre exemplaire; non plus que je ne viserai à résoudre sur le plan théorique les problèmes posés ici. Pour moi, le moment théorique serait plutôt l'intervalle, le point de passage entre l'examen de ma pratique passée et le problème toujours reposé de la poursuite de cette pratique. Ce que je cherche à résoudre, tant par des textes que par des films, et peut-être est-ce là une conception légitime de la théorie, c'est la problématique du fait de continuer à faire du cinéma.

J'accorderai une attention privilégiée, quoique non exclusive, au cinéma d'animation, d'abord parce qu'il s'agit de mon secteur de pratique, et aussi parce que la musicalité y prend un relief particulier. En effet, dans la mesure où la continuité temporelle même du film d'animation constitue une pure construction, fabriquée image par image, hors du temps et dans un état d'éparpillement discontinu, la musicalité est totalement coupée du domaine où la sonorisation naturelle (c'est-à-dire qui vise à 
reproduire la solidarité naturelle de la perception visuelle et sonore des phénomènes) peut avoir quelque pertinence. Même dans le cas limite où le choix est de mimer la sonorisation naturelle, cette dernière apparaît quand même, radicalement, comme une pure construction sonore, donc aspirée par la musique. Son caractère naturel est inévitablement de second degré. Ainsi, on peut dire que d'emblée, la musicalité est la tendance «naturelle» des trames sonores de cinéma d'animation, que celles-ci soient ou non musicales au sens strict.

Il ne s'agit encore que du rapport son-musique/image intérieur au cinéma. Mais le fait que, autant du côté de l'image que de celui du son, il s'agisse de quelque chose de construit, sans interférence avec le processus d'empreinte photographique ou magnétique des apparences visuelles et sonores de la réalité (processus qui est constitutif du cinéma de prise de vue réelle), crée un terrain où rien d'objectif (ou d'apparemment objectif) n'est acquis. La question des principes de construction s'y pose à visage découvert tant pour la partie visuelle que pour la partie sonore/musicale et, de là, celle de principes communs qui fonderaient l'unité des œuvres cinématographiques, et même, celle de l'importation des principes de I'un vers l'autre, notoirement de la musique vers le cinéma. Je ne veux pas dire ici que le cinéma de prises de vues réelles serait, quant à lui, clairement fondé sur un postulat de sonorisation naturelle, loin de là (nous le verrons), mais, simplement, que cette question du caractère construit et des principes de construction a, en cinéma d'animation, une netteté expérimentale. C'est dans les films d'animation abstraits qu'a été menée une grande partie des tentatives systématiques de «musique visuelle» qui néanmoins, il faut le dire, questionnent uniquement la musicalité de la bande image prise en elle-même et non la complexité de langages hétérogènes qui constitue ce qu'en général on désigne comme le «cinéma».

En l'occurence, signalons tout de suite le caractère polémique de cette définition musicale du cinéma. Dès l'époque du cinéma muet, et de façon constante depuis, la référence musicale a servi de contrepoids à la conception narrative du cinéma, la conception dominante, industrielle, d'un cinéma fait pour conter des histoires au grand public. Paradoxalement, cette référence musicale s'est souvent doublée d'une référence à la peinture et accessoirement à la danse comme si, au-delà de la démarcation face au cinéma narratif commercial, se constituait une sorte de front commun des arts qui prétendent aller plus loin que ce que la langue peut exprimer, face donc à tout ce qui a trait à l'expression verbale ou littéraire, étant entendu que la narrativité est assimilée au littéraire. Dans cette opposition, il est intéressant de noter que la sonorisation 
«naturelle» est le plus souvent associée au pôle narratif. Nous verrons que cette dichotomie a quelque chose de trompeur. Mais pour l'instant, nous allons parcourir sommairement l'histoire de chacun de ces pôles.

\section{La sonorisation «naturelle»}

Je commence par l'histoire de la sonorisation «naturelle» parce que c'est d'abord une histoire technique qui précisément met à jour le caractère flottant de la spécificité du cinéma. En effet, toute pensée de la spécificité du cinéma est nécessairement différente de celle de la peinture, par exemple, qui trouve à se définir en termes de gestes de la main dans leurs rapports avec la pensée et la vision, ce sans que l'histoire des données techniques n'y joue un rôle déterminant. Il s'agit toujours d'abord d'une histoire du sujet, de la pensée en acte par le corps, et de la société humaine. Il en va différemment du cinéma, qui est né d'une invention technique, ou plutôt d'une succession d'inventions techniques (d'abord la photographie puis le mécanisme d'entraînement du film, en passant par les multiples jeux optiques mettant à profit la persistence rétinienne) et dont la pratique comme art fut, jusqu'à ce jour, régulièrement bousculée et forcée à des redéfinitions par une histoire technique dont les ressorts fondamentaux ne sont pas artistiques, du moins pas dans le sens traditionnel du terme. L'histoire technique du son n'y échappe pas.

Le cinéma a d'abord été muet pendant près de 30 ans. Certains ont pu prétendre qu'il s'agissait là de la forme pure du cinéma du fait qu'elle n'était que visuelle et dynamique. On sait que cette pureté ne résiste pas à l'analyse : le cinéma n'était alors que technologiquement muet, les comédiens parlaient sans qu'on les entendent et en outre il y avait du son en présence réelle dans les salles de cinéma : des orchestres, un pianiste, des bonimenteurs qui commentaient et même parfois des comédiens qui donnaient des voix aux acteurs filmés. Il y avait les intertitres qui présentaient une contrainte cinématographique majeure, contrainte de nature éminemment littéraire. Donc, pas de pureté sinon celle qu'on s'est parfois donnée comme projet, telle l'avant-garde allemande sur laquelle nous reviendrons plus loin. On peut penser que toute ces pratiques étaient une simple anticipation de la venue au cinéma de l'invention de l'enregistrement sonore. À l'inverse, on peut tout aussi bien penser que le cinéma de cette époque restait incrusté dans une gangue théâtrale et que l'émancipation complète de cet art restait à venir. Il est paradoxal que ce soient les débuts du «parlant» qui aient été taxés de théâtraux alors que la présence 
réelle, fondement de la représentation théâtrale, venait d'être définitivement éliminée.

Quoi qu'il en soit, l'apparition du cinéma sonore a forcé une redéfinition fondamentale, souvent déchirante, de l'art cinématographique. Ce n'est qu'à partir de cette date qu'il devenait techniquement possible au cinéma de reconstruire la solidarité naturelle des perceptions visuelle et auditive des phénomènes. Cependant, longtemps la technique est restée lourde et laborieuse et ce n'est qu'à la fin des années 50 que l'enregistrement synchrone du son réel avec du matériel léger et autonome, lors des tournages documentaires, est devenue chose aisée, d'où une autre mutation profonde qui a marqué l'ensemble du cinéma. Plus récemment, sorte de retour du balancier, la généralisation du procédé Dolby stéréo ainsi que l'informatisation des consoles de mixage a laissé champ libre à une sorte d'hyperréalisme sonore totalement artificiel dont la reproduction spatialisée va souvent jusqu'à contredire les conventions d'espace liées à l'écran de cinéma; la valeur du hors-champ sonore, en particulier, s'en trouve profondément transformée.

De ces quelques éléments, on peut tirer la conclusion que l'idéal de réalisme sonore, la transparence son-image et le caractère d'évidence de leur lien intime, portés par le cinéma dominant, ne sont qu'une visée idéologique qui n'a jamais eu de base technique stable et qui a toujours été en décalage par rapport à la solidarité naturelle de l'ouie et de la vue. Une construction technologique largement marquée par des conventions. À cet égard, la présence non motivée de la musique dans les trames sonores n'est pas la moindre des conventions qui, d'ailleurs, n'a pas du tout la même valeur selon qu'il s'agisse du «muet» ou du «parlant». Dans le cas du «muet», la présence réelle des musiciens induisait un ordre de motivation dont même la présence des musiciens à l'écran n'est jamais l'équivalent.

Cette histoire est donc celle d'une équivoque qui pose comme naturel ce qui n'est qu'un possible technologique fortement surdéterminé par le fameux effet de réalité de l'image cinématographique. Face à l'affirmation d'une cohésion image/son fondée sur le naturel, l'autre histoire, qui n'en est pas l'opposé symétrique, est celle de la recherche de la pureté formelle d'un cinéma qui se définit d'abord comme visuel, mais qui cherche sa cohésion conceptuelle en prenant la musique comme modèle.

\section{Cinéma abstrait et musique}

On trouve la première expression à la fois théorique et pratique du «cinéma comme musique» chez l'avant-garde cinématographique allemande des années 20. Ces cinéastes, qui 
pour la plupart étaient d'abord peintres, emploieront indifféremment la terminologie de la peinture et celle de la musique, dans leurs textes théoriques comme dans les titres de leurs œuvres. Leur propos était de jeter les bases de l'autonomie de la création cinématographique face à la fonction de reproduction du cinéma, visant à réaliser des films absolus, selon le mot de Hans Richter. Ce mouvement de cinéma d'animation abstrait est né, avec un délai d'une dizaine d'années, dans la foulée de l'apparition de la peinture non figurative dont les protagonistes avaient déjà largement eu recours à des métaphores musicales pour défendre et illustrer l'autonomie et la pureté de la peinture.

La référence musicale jouait donc à deux niveaux différents. Premièrement, le cinéma abstrait en tant que peinture en mouvement, donc se déployant dans le temps, se prêtait aisément à des considérations musicales de rythmes, d'orchestration, de contrepoint, etc. Deuxièmement, et plus profondément, autant pour la peinture abstraite que pour ce cinéma qui en était un développement, la musique apparaissait comme un archétype, pure de toute référence représentative ou littéraire, une sorte de mathématique spirituelle applicable à tous les arts non verbaux.

Il n'est pas sans pertinence pour notre propos de souligner un lien originel entre l'histoire technique du cinéma évoquée plus haut et l'apparition de cette peinture et de ce cinéma préoccupés d'absolu, d'autonomie et de musicalité. En effet, l'invention de la photographie, premier jalon technologique du cinéma à venir, a constitué une intrusion majeure de la science, de la technologie et de l'industrie au cœur du processus de création, sous forme d'une première «machine» qui automatisait la vision. Elle a plongé la peinture dans une crise sans précédent. D'un coup, sa légitimité figurative, telle qu'elle s'était élaborée depuis la Renaissance et qui menait directement à cette invention, était anéantie et elle devait trouver ailleurs son fondement et sa spécificité. C'est une des raisons fondamentales de son évolution accélérée suite à ce choc, sorte de fuite en avant dont l'art abstrait a représenté un point extrême.

La polémique contre le cinéma commercial au nom de la pureté formelle dissimulait en réalité une autre bataille, plus profonde, des arts plastiques pour donner une nouvelle assise à leur légitimité, sur le terrain technologique même qui était à l'origine de leur désorientation. Le développement exponentiel de ces technologies de l'image a par la suite poussé jusqu'à l'extrême cette crise des images et de leur statut, qui ne s'est pas encore résolue. C'est dans ce contexte que l'idée de musicalité est apparue comme spiritualité et comme recours formel pour fonder une conception 
du cinéma qui transcende la fragilité mouvante de son assise technique, ainsi que le «commercialisme» toujours resté largement dominant. Un art confiant, engagé dans le progrès technique mais infiniment frustré de son territoire et ce, jusque dans l'art vidéo actuel.

L'école allemande abstraite des années 20 fut un épisode relativement bref qui prit fin avec la manifestation Der Absolute Film, qui eut lieu à Berlin en 1925. Cependant, Ruttmann, Eggeling et Richter apparaissent toujours comme les pionniers d'un courant qui, bon an mal an, a subsisté jusqu'à nos jours : que ce soit à travers Oskar Fischinger, qui visait explicitement la traduction cinématographique de pièces musicales connues, développant une sorte de syntaxe musicale du cinéma et qui a influencé nombre de cinéastes américains de la côte Ouest (dont les frères Withney), que ce soit à travers Len Lye et Norman McLaren, dont le rapport avec la musique était aussi fondamental mais plus libre.

C'est ici, dans la filiation directe de McLaren, que commence l'histoire de mes premiers démêlés avec la musique et la musicalité. $\mathrm{Au}$ début des années 60 , le format conventionnel des films d'animation abstraits était l'association d'une bande image et d'une pièce musicale. Peu importait que l'image ait été faite sur une musique préexistante, que la musique ait été composée d'après l'image ou qu'il s'agisse d'une association après coup de l'image et de la musique. En fait, à l'inverse de la recherche systématique d'un Fischinger, McLaren avait couvert librement, sans système et sans dogmatisme, tout l'éventail de ces possibilités (Rythmetic (1956), Mosaic (1965), Synchromy (1971) : musiques composées par McLaren - Begone Dull Care (1949) : film d'après la musique préexistente d'Oscar Peterson - Blinkity Blank (1955) : musique composée d'après les images par Maurice Blackburn Sphères (1969) : juxtaposition après coup de l'image et d'une pièce de Bach, jouée par Glen Gould), jugeant de la procédure selon l'éloquence du résultat.

Cependant, quelle qu'ait été la méthode de couplage, il y avait néanmoins toujours présomption d'une parenté profonde entre les deux versants visuel et musical, comme en témoigne le titre de ce documentaire britannique fait à son sujet, The Eye Hears, the Ear Sees (Gavin Millar, 1970). À ce que je sache, l'expression n'est pas de McLaren lui-même, mais elle est très symptomatique d'un refus, caractéristique de tout un courant de pensée, de fonder le cinéma sur le lien naturel entre la vue et l'ouïe, au nom d'un ordre supérieur, nécessairement abstrait ou formel, comme la musique, qui unifie les deux registres perceptuels au-delà du domaine du 
langage. Sur ce dernier point, le commentaire suivant de Guy Glover est révélateur d'une constante :

Par-delà le film parlant, ce domaine de la cinématographie qui délimite et parfois outrepasse celui du langage, il en existe un autre d'où la parole est presque exclue et qui est à la frontière de la musique et de la danse. C'est là que Norman McLaren, dont les films ne nous parlent que par l'image et le mouvement, s'est taillé une place au soleil (p. 9).

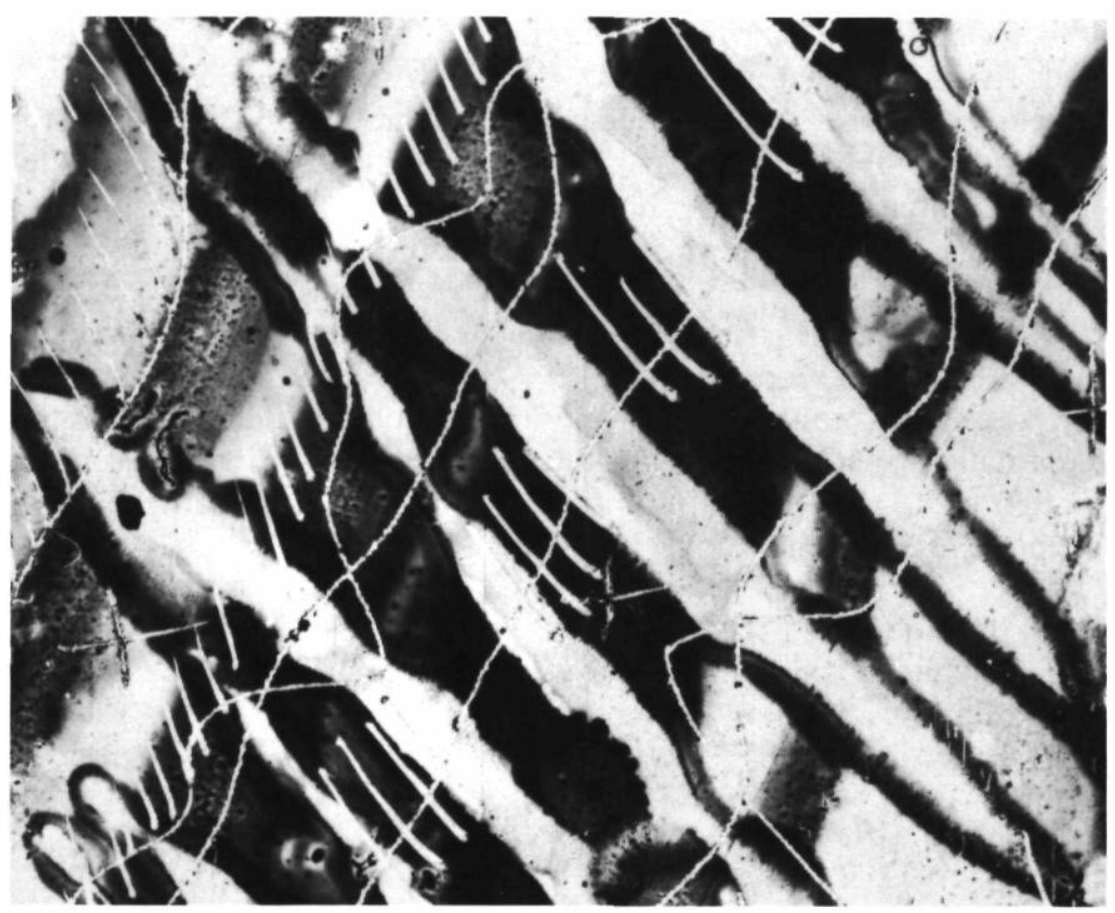

Begone Dull Care de Norman McLaren (1949) Coll.: ONF

Chez McLaren, l'exclusion à peu près totale de paroles ne tient pas nécessairement à une méfiance à l'endroit du langage vu comme carcan rationnel, comme c'est souvent le cas dans la rhétorique qui accompagne le «cinéma musical». Cette exclusion venait tout autant d'une intention généreuse, celle d'inventer une sorte de langage universel, dont ici encore la musique peut faire figure de modèle, qui parle à tous les peuples de la terre au-delà 
des barrières linguistiques. Il a réussi son pari, ses films ont eu une diffusion universelle et, comme on le dit de la musique, ont certainement contribué à adoucir les mœurs. Il l'a réussi, devraiton ajouter, dans les limites de son œuvre personnelle. En effet, vue sous un éclairage plus général, cette idée n'est pas sans poser certains problèmes, comme nous le verrons plus loin. C'est la question de Babel.

Pour l'instant, contentons-nous de souligner le fait que l'idée de musicalité va souvent de pair avec une volonté de dépasser les limites du langage, tant ses limites intrinsèques que celles qui se dressent entre les hommes. Cette mise en question du cinéma face au langage et du langage dans le cinéma, problématique double comme pour le rapport cinéma/musique, désigne également le passage du «muet» au «parlant» comme un moment charnière. Peut-être n'est-ce pas un hasard si les premiers films de McLaren datent de cette époque.

\section{4-71 - Mes premiers films}

C'est baigné par ce courant d'interpénétration diffuse de la musique et du cinéma que j'ai fait mes premiers films. Cependant, je n'ai pas abordé la question avec la sagesse et la modestie empirique de McLaren; j'étais un esprit plus dogmatique, influencé de façon plutôt éclectique par les courants artistiques les plus radicaux des années 60 dont, en arts plastiques, le op art, le minimalisme et l'art conceptuel, et, en musique, John Cage, Xenakis, le free jazz et surtout le sérialisme intégral qui florissait à l'époque. J'admirais les toiles d'Yves Gaucher dont l'argument était explicitement musical, et je m'essayais moi-même à faire des images calculées à partir de séries empruntées à des œuvres de Berg ou Webern. De 1964 à 1971, il en est résulté cinq films abstraits (Opus 1, 1964; Op hop, 1965; Opus 3, 1967; Autour de la perception, 1968; Notions élémentaires de génétique, 1971), tous inspirés d'une vision très systématique de la musicalité du cinéma.

Au premier abord, j'avais été tenté par la recherche de correspondances strictes entre les images et la musique, plus dans l'esprit de Fischinger que de McLaren. Ces recherches ont vite tourné court. Par exemple, cherchant, sans le support de son ou de musique, à produire l'effet d'un rythme à trois temps avec des moyens strictement visuels, je me suis rapidement rendu compte de l'impossibilité de l'entreprise. Seule l'audition simultanée d'une musique à trois temps permettait de percevoir l'image comme une valse. J'en ai acquis la conviction, que je n'ai pas remise en question depuis, qu'une même organisation métrique des durées prend une valeur totalement différente selon qu'elle se matérialise 
en images ou en sons, qu'il s'agit donc de réalités hétérogènes nécessitant des approches formelles totalement distinctes.

La conclusion que j'en tirai alors était néanmoins limitée dans sa portée : elle touchait strictement la possibilité de correspondances terme à terme entre la musique et les images cinématographiques. Laissant de côté le transfert direct de principes musicaux, je m'étais rabattu sur une vision plus abstraite qui consistait à mettre sur le même plan, dans le cadre d'une organisation formelle commune, les événements visuels et sonores/musicaux. Les sons et les images ne cherchaient plus à se refléter mais coexistaient, interagissaient dans une structure unique qui les transcendait par son idéalité. Il en résultait que je composais (ou plutôt bricolais) moi-même la musique, que je n'appelais d'ailleurs pas musique puisqu'il s'agissait d'une seule composition, à la fois visuelle et sonore. L'objectif de la pureté et de l'unité, conceptuelle dans ce cas, au-delà de l'hétérogénité des matières concrètes, sensibles, visuelles et sonores, restait total.

Ces expérimentations ont pris fin brusquement. Notions élémentaires de génétique m'a semblé sombrer dans un byzantinisme formel alors que les courants artistiques formalistes qui m'inspiraient commençaient eux-mêmes à s'essouffler. Pendant les 10 années suivantes, mon travail a été marqué par un usage spécifique et ponctuel de la musique. Père Noël, Père Noël (1974) est un exemple type de cette approche qui jouait sur le contraste entre une animation assez brute des personnages en papier découpé et une sonorisation réaliste très méticuleuse. L'écart était conscient et recherché. Je m'en expliquais par un recours à la théorie brechtienne de la distanciation. Mes sources d'inspiration étaient donc théâtrales et cinématographiques, dans la mesure où certains cinéastes comme Godard se situaient d'emblée dans ce courant de pensée. La musique était remise à sa place, définie en termes strictement dramaturgiques, mais elle n'avait quand même pas dit son dernier mot.

\section{3-92 - Rencontres avec des musiciens}

En 1983, avec Étienne et Sara, c'est le retour du refoulé : une nouvelle période d'expérimentation musicale qui dure depuis bientôt 10 ans et dont le terme n'est pas atteint. Contrairement à la période 1964-71, j'avais de fortes résistances à travailler de nouveau avec des trames sonores principalement constituées de musique. Ainsi, ce travail, très près de la musique et des musiciens, n'allait jamais avoir comme objectif la fusion formelle des images et de la musique, mais plutôt l'exploration de leur hétérogénéité. 
Cette deuxième aventure musicale a donc débuté et s'est poursuivie moins sur la base d'un intérêt pour la musique comme telle que de rencontres avec des musiciens : d'abord René Lussier, puis Robert M. Lepage et Jean Derome, puis finalement Fred Frith et Bob Ostertag, rencontres qui se faisaient sur le plan d'affinités tant personnelles qu'artistiques. Dans cette nouvelle perspective, qui mettait d'abord en rapport des créateurs différents engagés dans des disciplines distinctes vues comme incommensurables, le travail commun, l'interaction, ne pouvait avoir lieu qu'en termes d'échange et non de fusion, mettant en jeu non seulement la distance entre les disciplines mais aussi la distance entre les sujets.

Il faut tout de suite souligner une autre différence non négligeable : il ne s'agissait plus de films abstraits mais de films figuratifs. Peut-être est-il possible d'imaginer que des films abstraits puissent être conçus hors de la métaphore musicale, bien qu'historiquement il n'y en ait pas beaucoup d'exemples, mais chose certaine, des films figuratifs imposent un rapport à la narrativité, fût-elle non linéaire, et au langage qui limite la possibilité d'en poser l'organisation strictement en termes de formalisme musical. C'est tout un chapitre qui pourrait s'ouvrir ici au sujet du type de narrativité propre à l'animation gravée directement sur pellicule, qui se situe à une limite extrême de la cinématographie. Restons-en à cette remarque sommaire, car nous serions entraînés hors de notre sujet. Bref, le fil qui me rattachait à l'avant-garde allemande était quelque peu rompu.

Il est également à noter que ce qui m'a attiré chez ces musiciens, ce ne fut pas tant leur musique que leur façon de travailler. Je veux parler ici du lien que je trouvais, chez eux, entre, d'une part, leur pratique de l'improvisation, avec tout ce que cela implique d'interaction entre le corps et la musique et, d'autre part, leur usage du studio multipiste comme instrument de composition avec tout ce que cela implique d'incorporation de matériaux sonores de sources diverses, y compris extra-musicales. Chaque fois que j'étais témoin de scéances de travail, j'avais sous les yeux une malléabilité de la matière artistique et un rapport entre corporalité et technologie qui me faisait envie et qui me semblait correspondre profondément à ce que je cherchais dans mon propre travail. S'ensuivirent certaines conséquences formelles.

L'observation que j'avais déjà faite de l'incommensurabilité rythmique de la musique et du cinéma s'est approfondie et a pris, en quelque sorte, une valeur positive. J'ai, par exemple, pu observer à maintes reprises comment les musiciens, quand ils travaillent pour le cinéma, se plaignent de ne pas disposer d'assez de temps pour de véritables développements musicaux tels qu'ils les concevraient en dehors de la contrainte cinématographique. 
Paradoxalement, la musique, malgré une sensibilité au temps beaucoup plus grande que l'image cinématographique, s'avérait avoir besoin, pour exposer et développer une idée, de beaucoup plus de temps que l'image, dont la présence dans le temps jouit d'une sorte d'instantanéité. L'incommensurabilité des deux médias au sein du temps m'apparaissait alors déborder du niveau de la métrique pour englober désormais tout l'espace compositionnel, à grande et à petite échelle.

Du coup, la pratique courante en cinéma d'animation qui consiste à analyser image par image une pièce musicale pour pouvoir calculer le synchronisme des images et des sons m'est apparue profondément arbitraire. En outre, mon réflexe critique à l'endroit de l'idée même de synchronisme a trouvé confirmation dans certaines expériences faites sur scène, en particulier lors du spectacle The Technology of Tears (1987) qui fut une collaboration avec la chorégraphe Rosalind Newman et le musicien Fred Frith. Le problème s'est d'abord présenté comme un problème technique.

\section{La synchronisation flottante}

La présentation simultanée de la danse, de la musique et du film avait lieu dans des conditions techniques plutôt primitives si on les compare aux dispositifs de synchronisation par ordinateur utilisés par les spectacles dits «multi-média». Le film et le son étaient diffusés par des appareils distincts et non reliés; par conséquent leur vitesse de déroulement, relative, n'était jamais la même. Le début de la pièce était la seule marque de synchronisation fiable. Le film et la musique étaient donc en état de désynchronisation variable. Les danseurs faisaient leur possible pour suivre la musique, étant donc eux aussi dans un rapport variable avec le film. Cette situation de synchronisation flottante qui, initialement, apparaissait comme un sérieux handicap s'est finalement avérée une précieuse source d'expérimentation et d'enseignement.

Comme il fallait tout définir en termes de décalages et de relations à distance variable plutôt que de synchronisme, le film, la danse et la musique se trouvaient d'emblée placés chacun sur son terrain propre, dans un certain isolement par rapport aux autres composantes, et ce de façon plus marquée en ce qui concerne les images cinématographiques. À ma grande surprise, plutôt que de créer une situation floue et informe, cet état de flottement faisait apparaître des rapports plus complexes et plus riches entre la musique et le film, rapports qui, en outre, à l'intérieur de certaines limites, se renouvelaient d'une représentation à une autre. Chaque fois, de nouveaux aspects de l'image étaient révélés. Il m'est 
apparu alors que lorsque le synchronisme est mis au premier plan, au-delà de l'efficacité immédiate produite par la simultanéité des sons et des images, il s'ensuit un aplatissement des rapports images/musique, tous les autres rapports possibles étant masqués par le synchronisme.

Cette expérience m'a fait constater la mobilité particulière de l'animation gravée directement sur la pellicule par rapport à la musique. C'est un fait connu que la musique a un effet considérable sur la perception des images, tant sur le plan du climat émotif que sur le plan de la perception de leur organisation formelle. Ce phénomène est poussé à son état extrême dans le cas de la gravure sur pellicule. L'instabilité de l'image, d'un cadre à l'autre, fait que n'importe quel photogramme peut devenir un point de synchronisme éventuel avec un événement musical. La perception de la suite d'images s'organise autour de photogrammes pivots qui sont en fait désignés comme tel par la musique. Chaque rencontre différente avec la même musique, ou encore avec des musiques différentes, produit son propre effet spontané de synchronisme et ce, bien sûr, à l'intérieur d'un registre de potentialités déjà contenues dans une suite donnée d'images.

Pour faire face à ces situations de synchronisation flottante, j'avais quand même mis au point un petit système formel de cycles d'images. À chaque répétition du cycle, une image était abandonnée et une nouvelle ajoutée de sorte que deux métriques en décalage se superposaient, l'une fondée sur le point de reprise du cycle, l'autre sur les occurences successives de la même image diverses utilisations de ce principe de base sont à la source des différentes parties du film O Picasso (tableaux d'une surexposition) (1985). Ceci créait, à l'intérieur même de la suite d'images, une ambiguïté rythmique qui permettait d'absorber les décalages inévitables avec la musique très rythmée de Fred Frith et de garder une forte impression rythmique sans synchronisme calculé. $\mathrm{Ce}$ système de «cycles décalés» était d'un formalisme quasi musical. Tout en ne se justifiant pas d'une quelconque imitation de la musique, c'était tout de même une façon de tempérer les écarts. J'allais par la suite perdre ces scrupules.

L'exemple que je viens de commenter n'est pas tiré d'un film mais d'un spectacle. C'était anticiper un peu. Je dois d'abord souligner que cette vision «à distance» du travail avec les musiciens a déterminé un certain relâchement de mon rôle de réalisateur par rapport à la musique dans la série de trois films incluant Chants et danses du monde inanimé - Le métro (1985), Ô Picasso (tableaux d'une surexposition) et Adieu bipède (1987). Les musiciens (d'abord le duo Lepage-Lussier, puis le trio Derome-LepageLussier) y étaient pleinement responsables de la musique sans que 
j'y joue le rôle décisionnel qui est habituellement imparti au réalisateur.

L'expression «performance cinéma/musique» est inscrite au générique de ces oeuvres, faisant en cela référence au courant performance art. Cette désignation avait évidemment à voir avec le fait que tous ces films ont d'abord existé en spectacle, mais il s'agissait également d'indiquer que leur processus d'élaboration était déjà une "performance», une mise en scène des rapports cinéaste/musiciens qui débordait largement les moments de spectacle au sens strict. Il n'est donc pas étonnant que cette dislocation de la forme film ait impliqué un débordement de plus en plus important vers le spectacle vivant, où il n'était plus très clair s'il s'agissait encore de cinéma.

\section{«... faire comme un musicien»}

Dans un premier temps, avec La Symphonie interminable (1985), ces incursions dans le domaine du spectacle prirent la forme d'un rappel des pratiques sonores du cinéma muet avec cependant une volonté plus poussée de théâtralisation. Les films étaient projetés sans trame sonore et la musique exécutée en direct. $\mathrm{La}$ sollicitation du public sous le double registre cinématographique et théâtral constituait une dramatisation du processus à deux versants décrit plus haut. C'est cependant dans un deuxième temps, avec les spectacles d'animation gravée sur pellicule en direct, que mon rapport ambivalent avec la musicalité a pris une forme vraiment exacerbée.

Sans entrer dans les détails, il s'agissait de graver des images, par interventions cumulatives, sur une boucle d'amorce noire $16 \mathrm{~mm}$ pendant qu'elle tournait de façon ininterrompue dans un projecteur. La motivation qui m'avait amené à mettre ce dispositif au point était expressément «de faire comme un musicien», d'avoir à ma disposition une façon d'animer assez légère et rapide pour être l'objet d'une présentation en direct et pour que je puisse, à tout moment, m'associer à des musiciens dans des spectacles d'improvisation, plus ou moins sur le même pied d'égalité qu'eux. Lorsque pendant ces années, je fus amené à me produire dans des festivals de jazz ou de «musique nouvelle», c'est avec fierté que je portais le carton d'identification qui me désignait comme «musicien».

Tout ceci reposait sur cette même idée de synchronisation flottante exposée plus haut. Il s'agissait maintenant de beaucoup plus que de légers flottements. Le registre allait de la possibilité de points de synchronisation très précis, décidés néanmoins de façon indépendante par moi-même ou le musicien, jusqu'à des moments 
de fonctionnement totalement parallèles et autonomes de la musique et de l'image. La malléabilité de l'image à la musique s'y est vue mise à l'épreuve de façon constante. Ainsi, le projet $L a$ plante humaine a été confronté à plusieurs musiciens différents. Je citerai ici deux cas particuliers.

D'une part, il y a eu avec Robert M. Lepage un travail soutenu qui s'appuyait sur une collaboration déjà longue. Il en est résulté, de part et d'autre, la constitution d'un matériau stable déjà acquis qui donnait un cadre solidement établi aux nouvelles improvisations. Ce travail exploratoire se dirigeait en fait vers l'élaboration d'un film qui, dans l'avenir, devrait prendre une forme achevée. À l'inverse, il y a eu avec Bob Ostertag une série de collaborations ponctuelles à partir d'un univers musical très différent qui, pour moi, insufflaient de temps en temps un souffle nouveau au tronc commun de La plante humaine et qui, pour lui, à partir de la même matière filmique de base, ont servi de banc d'essai pour deux pièces complètement différentes : Sooner or later (endisqué sur étiquette RecRec Music - RecDec 37) et une composition pour quatuor à corde et bande magnétique commandée par le quatuor Kronos.

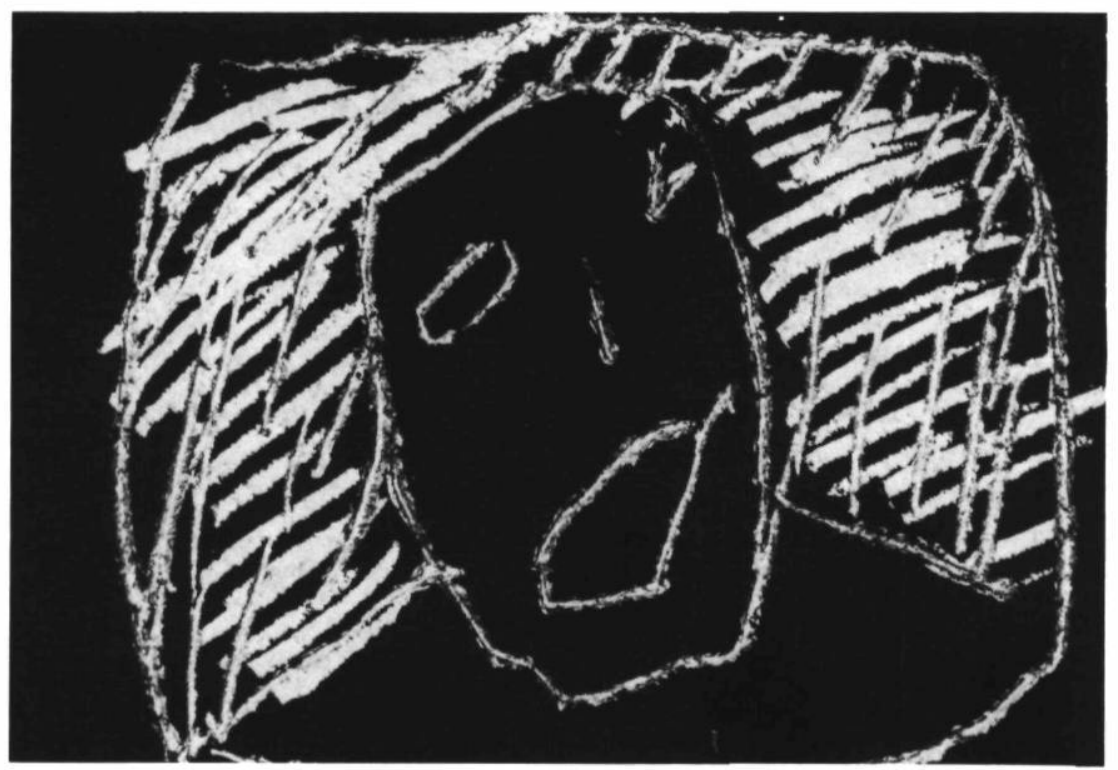

La Plante humaine de Pierre Hébert (l.m. en préparation) Photo : Pierre Hébert 
La diversité de ces confrontations de mes images avec la musique, ou plutôt avec des musiques, fut passionnante et féconde, car ce furent autant d'éclairages différents jetés sur la musicalité potentiellement contenue dans les images. Du coup, c'était aussi un éclairage différent qui fut jeté sur la problématique de la musicalité du cinéma, la faisant apparaître non comme un élément intrinsèquement musical dans le cinéma mais comme quelque chose qui serait donné au cinéma, ou plutôt qui serait révélé dans le cinéma par le face à face avec la musique, sans qu'il n'y ait rien de strictement musical d'importé dans le cinéma.

À la suite de ce que je viens d'exposer, on pourrait penser que finalement n'importe quelle musique pourrait faire apparaître un ordre de «musicalité» dans un film donné. Théoriquement cela est vrai, mais en pratique ce qui m'intéressait c'était de travailler la distance et non l'indépendance absolue. Alors, j'ai choisi de travailler avec certains musiciens bien précis avec lesquels je me sentais des affinités et non avec d'autres. N'importe quelle musique n'aurait pas fait l'affaire. Ces affinités étaient personnelles et esthétiques, j'avais de l'estime pour les personnes et pour leur musique. Mais il fallait qu'il y ait davantage pour s'engager ensemble sur la corde raide.

Ce «plus», j'en suis venu peu à peu à le désigner comme une «affinité compositionnelle» sans toutefois être totalement en mesure de saisir la portée de cette expression. Je cherchais ainsi à situer l'élément commun qui rendait la rencontre possible, au-delà de ce qui fait que la musique et les images ont des façons irréductibles d'occuper le temps, sans pour autant retourner à la vision formelle de mes expériences des années 64-71. Au contraire, je voulais assumer la dimension formelle en autant qu'elle plonge ses racines d'une part dans la reconnaissance de la matérialité et de la technicité spécifiques de chaque discipline et, d'autre part, dans la subjectivité des deux artistes, à commencer par l'acceptation d'entrer dans la relation à distance décrite plus haut. Ouverture à l'autre et rejet de la coupure entre l'organisation formelle matérielle et la pensée (au sens de globalité du fonctionnement de l'esprit humain et non uniquement de pensée rationnelle), entre forme et contenu. À cette échelle, à cette distance devrais-je dire, il me semblait y avoir une zone dont la configuration et les limites ne seraient jamais données d'avance et où l'idée d'une musicalité du cinéma pourrait avoir un contenu positif.

Pour éclairer ce que j'espérais de cet étrange rapport avec la musique, il est utile de souligner qu'au cours de la même période, j'ai également recherché avec la danse, la peinture et l'écriture une 
semblable relation qui me permette, sous différents angles, une vue différentielle du cinéma ou, en tout cas, de ma position dans le cinéma. Mon travail des récentes années avec la musique a eu comme arrière-plan une pratique beaucoup plus large de l'interdisciplinarité, qui posait donc plus globalement pour moi la question de la spécificité et des rapports possibles entre différentes disciplines artistiques.

\section{Cinéma, musique et oralité}

La question étant ainsi posée plus largement, il est temps de revenir sur la problématique du langage, que j'ai effleurée à quelques reprises au cours de ce texte. Il est habituel que les arts non verbaux, dont la musique et le cinéma, se définissent volontiers comme un au-delà du langage articulé, considéré comme une forme de communication étroite, rigide et abusivement rationnelle. Le cinéma «musical», on l'a vu, se réclame volontiers de cette argumentation. Je ne vais pas mettre en doute ici le fait que cinéma et musique soient spécifiques par rapport au langage et que de ce fait ils expriment des choses qui, au sens strict, ne peuvent être «dites». C'est une différence qui est d'une nature similaire à celle qui sépare la musique et l'image cinématographique. Cependant, l'idée que l'ensemble des arts non verbaux dans une démarcation commune à l'endroit du langage s'adressent directement à une zone plus profonde de l'humain m'apparaît suspecte. Il me semble que le langage occupe effectivement une position singulière dans l'esprit humain par rapport aux autres formes de «communication», mais pas celle-là.

Pour éclairer cette question, je voudrais rappeler la conception de l'origine du langage développée par le paléontologue et archéologue André Leroi-Gourhan dans son ouvrage Le geste et la parole. L'apparition du langage y est vue comme un effet du développement de la station debout chez les hominiens, ayant pour conséquence bio-mécanique la libération simultanée de la bouche et des membres antérieurs. L'une fut libérée de ses fonctions de préhension et, les autres, de leur fonction de locomotion, permettant ainsi le développement parallèle du langage et de la production d'outils. Il en résulte que la capacité langagière de l'espèce humaine est indissociable du développement de la pensée et a joué un rôle pivot comme contrepartie de l'activité technique et, devrait-on dire, de toutes les autres activités productrices de sens, dont les arts figuratifs et musicaux. En d'autres termes, pas de peinture, de musique, ni, ultimement, de cinéma sans capacité langagière à la base. C'est en tant qu' animal doté du langage que l'homme peut créer du sens de toutes sortes de façons. 
On trouve chez le linguiste Émile Benveniste des indications intéressantes à cet égard. Il part d'une conception similaire à celle de Leroi-Gourhan :

Car l'homme n'a pas été créé deux lois, une fois sans le langage, et une fois avec le langage. L'émergence de Homo dans la série animale (...) est due avant tout à sa faculté de représentation symbolique, source commune de la pensée, du langage et de la société (tome 1, p.27).

Puis, il singularise le langage par sa position particulière dans l'ensemble des systèmes sémiotiques. Partant du principe de nonredondance («Il n'y a pas de "synonymie" entre systèmes sémiotiques; on ne peut pas "dire la même chose" par la parole et par la musique qui sont des systèmes à base différente» (tome 2, p. 53), il voit quand même la possibilité de rapport d'interprétance entre systèmes sémiotiques différents («... il s'agit de déterminer si un système sémiotique donné peut s'interpréter par lui-même ou s'il doit recevoir d'un autre système son interprétation» (p. 53) arrivant ainsi à la constatation que «les signes de la société peuvent être intégralement interprétés par ceux de la langue, non l'inverse» (p. 54 ).

Cette théorie du langage, si on l'accepte, fait apparaître notre discussion sous un jour nouveau et lui pose des questions fondamentales. Ainsi, à la lumière de ce qui précède, qu'en est-il de ce rapport fusionnel direct entre musicalité et cinéma qui, comme on l'a vu, s'exprime à peu près toujours sur fond d'une certaine méfiance à l'endroit du langage articulé? De quelle nature est ce rapport qui semble incontournable (y compris pour moi qui y résiste) et comment le situer face au rôle unique joué par le langage?

Ici l'hypothèse d'un «au-delà» ou d'un «plus profond» que les «mots» est d'emblée exclue dans la mesure où rien de ce qui constitue l'esprit humain et la vie sociale ne peut exister sans le langage. Encore faut-il lever une autre équivoque dont s'alimente la méfiance citée plus haut à l'endroit du langage. Celle-ci prend sa source dans une conception du langage où la réalité semble enfermée de force dans les petites boîtes-mots qui sont rangées dans des dictionnaires où leur sens est fixé d'avance et qui sont mis en relation selon les règles rigides de la rationnalité grammaticale, dans le but premier de servir à la communication utilitaire. À l'opposé on peut penser, à la suite de Benveniste, qu'au moment de l'énonciation, chaque fois que quelqu'un parle, le sens des mots s'ouvre de nouveau et que dans cette ouverture s'exprime toute la profondeur corporelle et psychique du locuteur ainsi que sa 
socialité et son historicité. Ce qui est en jeu alors, et qui se produit dans n'importe quelle élocution, même la plus modeste, est beaucoup plus que la simple communication conventionnelle d'un contenu par une forme.

Il faudrait citer à ce sujet la longue et patiente critique menée par Henri Meschonnic à l'endroit du dualisme du signe, contestant entre autres l'opposition couramment faite entre «langage ordinaire» et poésie, opposition qui place la poésie dans une situation d'écart par rapport au «langage ordinaire» et à la prose, comparable à celle revendiquée dans le cinéma au nom de la musicalité. Les travaux de Meschonnic culminent dans une notion de rythme «reconnu comme mouvement du sujet dans son discours, et pris comme subjectivité, historicité radicales du discours» (1991: p.11), qui se démarque résolument de la conception métrique dominante. $\mathrm{Ce}$ qui peut aider à comprendre pourquoi une approche strictement métrique du rythme ne permet pas de rendre compte, sinon de façon réductrice, de la coexistence dans le temps du cinéma, de la musique et de la danse. Dans ses études sur la versification, Meschonnic considère la conception métrique du rythme comme spécifique à la musique et inapplicable comme telle à la littérature. Cependant, l'introduction des synthétiseurs à métrique absolue a montré que même la musique ne peut se satisfaire d'une telle vision désincarnée et désubjectivée du flot temporel. La conception du rythme développée par Meschonnic pourrait être féconde pour la question débattue ici, si on la développe en tenant compte de la matérialité et de la technicité propres aux disciplines concernées.

Du point de vue de leur relation réciproque, le cinéma et la musique ont intérêt à une telle réhabilitation du langage. Peut-être serait-il possible d'imaginer alors que cette ouverture constamment recherchée par le truchement de la musicalité, face aux formes figées de la narration naturaliste, a quelque chose à voir avec l'oralité? C'est une hypothèse qui m'attire, car elle rejoint les termes avec lesquels je m'explique à moi-même ce que je fais quand j'anime. Quand, dans ma tête, je me dis : «J'essaye d'animer comme on danse» ou «... comme on fait de la musique» ou «...comme on peint» ou même «... comme on écrit», etc., toutes ces expressions sont englobées avec des tonalités différentes dans une seule affirmation : «Je veux animer comme je parle». Pour décrire mes collaborations étroites avec des musiciens, j'ai spontanément utilisé le terme «conversation» que j'ai d'ailleurs repris comme titre d'une série de performances impliquant du cinéma, de la musique, de la danse et de l'écriture (avec Robert M. Lepage, Louise Bédard et Sylvie Massicotte). 
Comme je l'ai expliqué plus haut, ce qui m'a attiré chez les musiciens, en 1983, et chez les danseurs un peu plus tard, n'avait pas tant à voir avec des rapprochements formels dans la façon d'organiser le temps ou le mouvement, mais avec le caractère matériel et charnel de leur activité. Le «temps» des musiciens et «les mouvements» des danseurs m'ont intéressé, non comme des catégories abstraites dans lesquelles je pouvais également situer ma propre activité d'animateur, mais comme temps et mouvement incarnés par d'autres dans le cadre de contraintes matérielles et techniques spécifiques et datées dans l'histoire particulière de ces disciplines. En cela, la danse et la musique dans leur rapport différentiel avec le cinéma ont constitué pour moi une expérience de l'altérité qui pointait le doigt vers le langage et l'oralité.

La question ne se réduit pas à savoir s'il y a ou non des paroles dans un film. L'idée, courante dans les cercles du cinéma d'animation, que l'absence de parole dans un film donne accès à l'universalité, m'apparaît douteuse, comme je le soulignais plus haut par rapport à McLaren. Que les films soient muets facilite indéniablement les choses d'un point de vue technique, sans plus. Mais peut-être que, du même coup, s'exprime une utopie babelienne qui rejette l'expérience fondamentale de l'altérité que constitue la multiplicité des langues. Je préfère penser que le succès universel des films de McLaren est plutôt attribuable au fait qu'ils sont profondément incarnés, marqués par une oralité fondamentale et par conséquent par la propre langue du cinéaste même s'il n'y a pas de parole.

Cette vision de la signifiance comme corporéité et matérialité tire la création artistique hors de l'opposition corps/esprit et, de ce fait, permet une approche différente de ce qui apparaît autrement comme une hégémonie de l'histoire technologique sur la définition du cinéma. Ce qui permet de poser différemment la double histoire que nous avons racontée au début de ce texte. En effet, la «volatilité» de la définition technologique du cinéma, qui est de nos jours aggravée par la porosité de ses démarcations avec la vidéo et l'image informatique, prend une valeur différente si elle est vue comme histoire de rapports indissociables entre corps, langage et technique.

J'ai entrepris ailleurs d'explorer ces questions (articles dans 24 images, dans La Revue de la Cinémathèque et dans Les Vendredis $d u$ corps, livre collectif à paraître). Je me contenterai donc d'ajouter que c'est un enjeu de notre époque que de définir la juste distance entre le langage et le corps, et pas seulement du point de vue de l'art mais aussi de la technologie, que l'idéologie dominante tend actuellement à présenter comme un facteur autonome et 
déterminant au sein de cette relation qui seule rend possible la pensée et l'action de l'humanité dans l'histoire. De ce point de vue, la responsabilité des artistes est grande de considérer la pensée et l'expression de cette juste distance comme partie intégrante de leurs œuvres.

Tout cela est au cœur de notre sujet de départ, la musicalité du cinéma, car les conditions mêmes qui permettent initialement de poser ce problème, tout comme celui, plus général, de la coexistence dans le cinéma de pratiques artistiques distinctes, viennent du développement technologique et varient à son gré. D'où une différence profonde entre l'opéra et le cinéma qui, tous deux, se fondent sur la coexistence des arts : la présence ou l'absence des corps. Lorsque la coprésence réelle des corps est rendue caduque par la magie de la technologie, la corporéité doit trouver un autre chemin. C'est ce qui m'apparaît être le problème premier de l'art aujourd'hui, et l'oralité au sens où nous l'avons défini est en son centre. À partir de ceci, il ne me reste qu'à cesser de parler et à essayer de faire un film.

\section{Office National du Film du Canada}

\section{OUVRAGES CITÉS}

Benveniste, Émile. Problèmes de linguistique générale, 1 et 2. Paris : Gallimard, 1988.

Glover, Guy. «Avant-propos». McLaren. Montréal : Office national du film, 1980.

Hébert, Pierre. «Les enjeux de l'art à l'ère des machines. Effacement et résistance du corps». 24 Images 43 (été 1989) pp. 22-27.

Hébert, Pierre. «Un cinéaste face aux nouvelles technologies». La Revue de la Cinémathèque (déc. 1989 - janv.1990) pp. 9-10.

Hébert, Pierre. "Entre la nostalgie du dessin et le désir de la danse». Esquisses psychanalytiques 17 (hiver 1992) pp. 189-202.

Hébert, Pierre. "Égarements volontaires». Les Vendredis du corps. Montréal : Jeu / Parachute, 1992 (à paraître).

Leroi-Gourhan, André. Le Geste et la parole. Paris : Albin Michel, 1964.

Meschonnic, Henri. Le Signe et le poème. Paris : Gallimard, 1975.

Meschonnic, Henri. Critique du rythme. Paris : Verdier, 1982.

Meschonnic, Henri. La Rime et la vie. Paris : Verdier, 1989.

Meschonnic, Henri. «Le langage comme défi». Le langage comme défi. SaintDenis : Presses universitaires de Vincennes, 1991, pp. 9-15.

Ropars-Wuilleumier ${ }_{2}$ Marie-Claire. «L'image dans le langage (Écriture filmique et théorie de la littérature)». Le langage comme défi. Saint-Denis : Presses universitaires de Vincennes, 1991, pp. 149-158. 